\title{
El papel de las competencias emocionales parentales en las conductas internalizantes y el autoconcepto de los niños
}

\section{The role of emotional parental competencies in children's internalizing behaviors and self-concept}
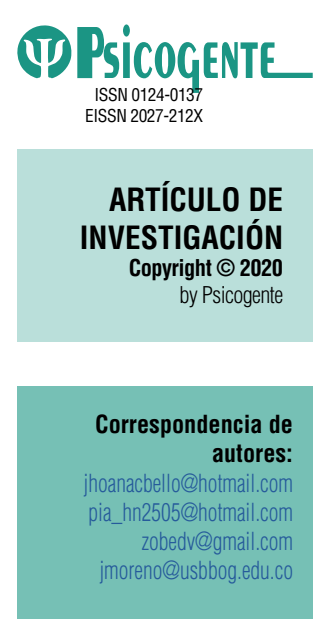

Recibido: $21-01-20$ Aceptado: $15-05-20$ Publicado: 01-08-20

\author{
Jhoana Carolina Bello Pulido (iD - Piedad Rocio Hurtado Nieto iD - Zobed Elena \\ Villalba Yibirin (iD - Jaime Humberto Moreno Méndez iD \\ Universidad de San Buenaventura, Bogotá, Colombia
}

\section{Resumen}

Introducción: Las competencias emocionales de los padres se constituyen en un factor protector para el fortalecimiento del autoconcepto en los niños; sin embargo, cuando no se expresan adecuadamente pueden generar en los hijos problemas internalizantes. La evidencia empírica en Colombia sobre la relación entre dichas variables no ha sido suficiente por lo cual es fundamental continuar su estudio.

Objetivo: Identificar la relación entre competencias emocionales de los padres, el nivel de autoconcepto y las conductas internalizantes en niños de 8 a 12 años.

Método: En esta investigación correlacional se aplicó el Cuestionario de Evaluación de Estilos Educativos de Padres (CEEP), la Lista de Chequeo de la Conducta Infantil (CBCL) - formato para padres; y la Escala de autoconcepto de Piers-Harris a los niños. Los participantes fueron 364, conformados por 182 diadas (padres e hijos de 8 a 12 años) de colegios públicos y privados de Bogotá, seleccionados bajo un muestreo no probabilístico por conveniencia.

Resultados: Se encontró una relación positiva estadísticamente significativa entre la impulsividad en padres y los síntomas ansioso/depresivo $(R h o=0,213 ; p=0,04)$ y aislado/depresivo $(R h o=0,210$ $p=0,004)$ en los niños. Se hallaron relaciones inversas entre el manejo emocional y el componente intelectual del autoconcepto $(R h o=-0,148 ; p=0,046)$, entre los síntomas ansioso/depresivo y los componentes conductual (Rho $=-0,240 ; p=0,001)$, físico $(R h o=-0,182 ; p=0,014)$ y falta de ansiedad de autoconcepto ( $R h o=-0,213 ; p=0,004)$.

Conclusiones: Las relaciones encontradas entre impulsividad y síntomas internalizantes en los niños, evidencian que los enfados excesivos e injustificados de los padres, pueden asociarse con preocupación excesiva y aislamiento social en los hijos. Estos hallazgos permiten sugerir para futuros estudios, evaluar el efecto de programas para prevenir problemas de ansiedad y depresión en los niños a través del entrenamiento en regulación emocional a los padres.

Palabras clave: Competencias emocionales, autoestima, síntomas internalizantes, ansiedad, depresión.

Abstract

Introduction: The emotional competences of the parents are a protective factor for strengthening the self-concept in children; However, when they aren't expressed properly, they can generate internalizing problems in the children. The empirical evidence in Colombia on the relationship between these variables hasn't been sufficient, so it is essential to continue its study.

Objective: To identify the relationship between parents' emotional competences, the level of self-concept and internalizing behaviors such as anxiety, depression and somatic complaints in children.

Method: In this correlational research, the Parent Educational Style Assessment Questionnaire (CEEP), the Child Behavior Check List (CBCL)- parent format and the Piers-Harris Self-Concept Scale were applied to children. The participants were 364 made up of 182 dyads (parents and children from 8 to 12 years old) from public and private schools in Bogotá, selected under a non-probabilistic sampling for convenience. Results: A statistically significant positive relationship was found between impulsiveness in parents

Cómo citar este artículo (APA):

Bello Pulido, J., Hurtado Nieto, P. R., Villalba Yibirin, Z. E. \& Moreno Méndez, J. H. (2020). El papel de las competencias emocionales parentales en las conductas internalizantes y el autoconcepto de los niños. Psicogente 23(44), 1-23. https://doi.org/10.17081/psico.23.44.3824 
and anxious/depressive symptoms ( $R$ ho $=0,213 ; p=0,04)$ and isolated/depressive $(R h o=0,210 ; p=0,004)$ in children. Inverse relationships were found between emotional management and the intellectual component of self-concept ( $R h o=-0,148 ; p=0,046)$, between anxious/depressive symptoms and behavioral components (Rho $=-0,240 ; p=0,001)$, physical (Rho $=-0,182 ; p=0,014)$ and lack of self-concept anxiety (Rho=-0,213; $p=0,004$ )

Conclusions: The relationships found between impulsivity and internalizing symptoms in children show that excessive and unjustified anger of parents can be associated with excessive concern and social isolation in children. These findings allow us to suggest for future studies, to evaluate the effect of programs to prevent anxiety and depression problems in children through training in emotional regulation for parents.

Keywords: emotional competences, self-esteem, internalizing symptoms, anxiety, depression.

\section{INTRODUCCIÓN}

El estudio del desarrollo infantil implica considerar la relación de múltiples variables, muchas de ellas vinculadas al contexto que rodea a los niños, niñas y adolescentes, tales como los patrones culturales, las características ambientales, los estilos parentales y el entorno familiar. En este sentido, es pertinente resaltar que la familia está compuesta por una serie de factores generadores de modelos e interacciones significativas donde se establecen prácticas educativas que se convierten en punto de referencia de estilos de socialización que permiten en los niños la adquisición de estrategias de afrontamiento y formas de interpretar el mundo (Ortiz, Sánchez, Rebollo, \& Etxebarria, 2014).

Las competencias parentales dentro del marco de la crianza de los hijos son consideradas como el conjunto de conductas que proporcionan el bienestar psicológico y el desarrollo íntegro de los niños, al tener en cuenta el afecto, protección, cuidado y orientación necesaria para facilitar dicho proceso en los distintos ámbitos de la vida (familiar, académico, social y comunitario) (López, Quintana, Casimiro, \& Chávez, 2009). De acuerdo a ello, un grupo de competencias cuyo papel en la crianza de los niños es sumamente relevante, son las emocionales, las cuales hacen referencia a las habilidades de los padres en el reconocimiento, expresión, el manejo de emociones y de la impulsividad (Gómez, García-Barranco, De las Heras, Martínez de Salazar, Cobos, Martín \& Fernández, 2013).

Como se refleja en dicha definición, tales competencias parentales son fundamentales para el ajuste de sus hijos, lo cual tendría que ver con el papel socializador de los padres en la crianza de los niños, ya que en medio de la interacción entre estos se ven implicadas una serie de acciones afectivas, disciplinares y educativas realizadas por los padres y agrupadas bajo el término "socialización" (Jiménez, 2015).

Denham, Bassett y Wyatt (2007) afirman que los padres son las principales figuras para modelar la expresividad emocional, enseñar sobre una variedad 
de emociones y reaccionar de forma contingente a estas, lo cual hace posible que estos aspectos de la socialización de la emoción se asocien y/o predigan la competencia emocional de los niños. Además, Ruvalcaba, Gallegos, Flores y Fulquez (2013) mencionan que la capacidad de cada persona para manejar sus emociones favorece la salud mental al prevenir la presencia de ansiedad o depresión.

En razón a lo anterior, el conocimiento y manejo de las emociones de los padres son un pilar importante para contribuir a la salud mental de sus hijos, ya que la deficiencia de competencias emocionales en el marco de la crianza pueden derivar en la dificultad de los niños para desarrollar habilidades que permitan afrontar las distintas situaciones vitales, con la probabilidad de que se generen problemas a nivel afectivo y comportamental, así como en su autoestima y autoconcepto (Moreno-Méndez, Muñoz, Sánchez, Triana, Chemas, \& Barón, 2011; Moreno-Méndez, Umbarilla-Contreras, \& FrancoTorres, 2019) y traumas infantiles que también se encuentran relacionados con la presencia de conductas de tipo internalizante (Vanegas, 2014).

Un aspecto fundamental en el desarrollo de los niños es el fortalecimiento de su autoconcepto, dado que favorece el sano desarrollo de su personalidad y la construcción efectiva de su propia identidad. Para tener un autoconcepto adecuado es indispensable un buen funcionamiento a nivel personal y social, debido a que el entorno contribuye en gran medida a que los niños tengan una imagen positiva de sí mismos (Esnaola, Goñi, \& Madariaga, 2008; Hincapié, Montoya, \& Dussan, 2016).

La autovaloración positiva o negativa de los niños puede estar relacionada con la presencia de problemas del comportamiento (Mazadiego, Vera, \& Ruiz, 2011). Las alteraciones de tipo emocional y conductual suelen ser muy comunes en niños menores a 10 años, de las cuales la presencia de conductas de tipo externalizante e internalizante son las más frecuentes. Estas últimas se caracterizan por su contenido especialmente emocional e incluyen síndromes específicos como la ansiedad, depresión, retraimiento y quejas somáticas (Hewitt-Ramírez \& Moreno-Méndez, 2018; López-Soler, Alcántara, Fernández, Castro, \& López Pina, 2010; Posada, 2013; Restrepo \& Rivera, 2017; Symeou \& Georgiou, 2017).

En la literatura existen distintos estudios que dan evidencia acerca del papel del proceso de socialización y de las competencias emocionales empleadas por los padres sobre las conductas internalizantes y el autoconcepto. Algunos autores reportan que los padres que se distinguen por falta de afecto o apoyo emocional y poca disciplina tienden a percibir un mayor retraimiento, altera- 
ciones emocionales y comportamentales en sus hijos (Franco, Pérez \& De Dios, 2014; Jadue, 2003; Martínez \& García, 2012). En la misma medida, se ha encontrado en la literatura que los niveles más altos de expresividad negativa en los padres se asocian con la regulación poco manejable y con los comportamientos no deseables de sus hijos, a su vez, la devaluación y castigo de las emociones negativas de los niños por parte de los padres dificulta la expresividad emocional (Cuartas, Ward, Ma, \& Grogan-Kaylor, 2020; Leathers, Spielfogel, Geiger, Barnett, \& Voort, 2019; Milojevich y Haskett, 2018; Mirabile, 2014; Sher-Censor, Shulman, \& Cohen, 2018).

Por otro lado, Ajilchi, Kargar y Ghoreishi (2013) concluyeron que el nivel de estrés materno está relacionado con la autoestima en los niños. En la misma medida, Paulus, Licata, Gniewosz, \& Sodian (2018) encuentran relación positiva entre el autoconcepto social de los niños y aspectos de la relación madre-hijo, como la sensibilidad materna y la no hostilidad.

Turcule y Tulbure (2014) exponen que la inteligencia emocional de los padres tiene un papel decisivo en el desarrollo emocional de los hijos, de este modo, si se trabaja la educación emocional de los adultos se pueden obtener resultados favorables en la educación y comportamiento de los hijos. Doan, Son, y Kim (2018) encuentran asociación entre la tolerancia a la angustia y la depresión en niños de 8-12 años, donde las estrategias de regulación de la emoción materna son un correlato importante.

Por su parte Prooijen, Hutteman, Mulder y Laceulle (2018), expresan que cuando los padres son compresivos, responsables y empáticos hacia las emociones de sus hijos, se refleja una disminución en la probabilidad de que el niño presente problemas de salud mental de tipo internalizante.

Al hacer una aproximación al estado de salud mental actual, se observa que en Colombia la cantidad de personas de 0 a 19 años que son atendidas por trastornos mentales y comportamentales incrementa cada día. Las estadísticas indican que de 2009 a 2016 se atendieron 1.827 .955 niños diagnosticados con código CIE 10 F00-F99 (trastornos mentales y del comportamiento), de los cuales se señala una mayor prevalencia de conductas internalizantes en edades comprendidas entre los 5 a 14 años, donde la depresión de tipo moderado representa el trastorno mental que más afecta a los niños y que a su vez produce mayor discapacidad (Ministerio Nacional de Salud Mental, 2017).

En coherencia con dichas estadísticas, no es posible ignorar el impacto que puede tener la presencia de trastornos en el ajuste personal, social, escolar, 
de la vida del niño. La Organización Mundial de la Salud (2017) y el Ministerio de Salud Mental (2017) reportan que la presencia de trastornos de salud mental, cuyos efectos se extienden a la salud general y el desarrollo del niño, tienden a ser un factor de riesgo de distintos problemas sanitarios y sociales en un futuro, como lo es el consumo elevado de alcohol, tabaco, sustancias psicoactivas, el embarazo adolescente, el abandono escolar y la manifestación de conductas delictivas.

Al tener en cuenta que desde la etapa infantil se pueden presentar diferentes trastornos que afectan el desarrollo del niño, relacionados con alteraciones de tipo emocional y del comportamiento que suelen ser muy comunes en niños menores de 10 años, como lo afirman Restrepo y Rivera (2017), y al identificar que los problemas internalizantes comienzan a manifestarse desde esta etapa de la vida, resulta pertinente estudiar el entorno en el que el niño se desenvuelve y donde pueden configurarse distintos factores protectores y de riesgo.

El presente estudio aportaría información relevante que responde al interés del campo de la psicología clínica, en la medida que permite indagar desde una perspectiva ecológica, las conductas internalizantes y las condiciones en las que se relacionan con las competencias emocionales parentales, lo cual servirá para el desarrollo e implementación de iniciativas de intervención a fin de disminuir la presencia de problemas de salud mental en la niñez.

Las implicaciones a nivel sociocultural son significativas al considerar que, si se promueven estrategias en pro del desarrollo de competencias emocionales parentales adecuadas podrían constituirse en un marco que propicie en los padres un cambio en el manejo emocional impulsivo y maltratante del castigo en la crianza que se ha transmitido culturalmente de generación en generación en el contexto colombiano, tal como se ha reportado previamente (Cuartas et al., 2020). A su vez, se contribuiría al bienestar socioemocional de los niños y al fortalecimiento del autoconcepto lo cual podría favorecer la sana convivencia y el ajuste social, personal, académico, y familiar del niño. También se fortalecería el establecimiento de vínculos con adultos o pares (Ternera, 2014).

La presente investigación se llevó a cabo en colegios públicos y privados de estratos bajos, debido a que se ha encontrado que el bajo nivel socioeconómico se encuentra relacionado con el desarrollo de problemas internalizantes en niños que asisten a colegios públicos, sin embargo, en algunos privados también se ha hallado, pero en otros no se ha encontrado (García, Espinosa, Jiménez, \& Parra, 2013; Ramírez, 2005; Vinacur, 2015). Al tomar en 
consideración lo anterior, se planteó como objetivo en la presente investigación establecer la relación entre las competencias emocionales parentales, conductas internalizantes y autoconcepto en niños de 8 a 12 años.

\section{MÉTODO}

\subsection{Diseño}

De acuerdo con la línea cuantitativa, con un paradigma empírico-analítico, se adoptó un tipo de estudio transversal y un método correlacional, al buscar determinar la relación entre las variables (en mención) en un momento temporal específico (Ato, López \& Benavente, 2013).

\subsection{Participantes}

La muestra estuvo compuesta por 364 participantes, conformados en 182 díadas de padres e hijos, entre 8 y 12 años, de colegios públicos y privados de la ciudad de Bogotá, bajo un muestreo no probabilístico, por conveniencia. Como criterios de inclusión se estableció que los niños debían ser residentes de Bogotá, tener edades entre 8 y 12 años, estar escolarizados y convivir por lo menos con uno de los padres. Los padres debían tener un nivel mínimo de escolaridad de quinto de primaria.

En la Tabla 1 se reflejan los datos sociodemográficos por parte de los niños. El rango etario más frecuente fue de 8 a 10 años con un $78 \%$, predominando el sexo masculino en un $51,6 \%$. Para el tipo de colegio el $64,8 \%$ pertenece a público y el $35,2 \%$ de la muestra pertenece a privado.

Tabla 1.

Datos sociodemográficos de Niños

\begin{tabular}{cccc}
\hline & & FRECUENCIA & PORCENTAJE \\
\hline \multirow{2}{*}{ Edad } & $8-10$ & 142 & 78 \\
& $11-12$ & 40 & 21,9 \\
Sexo & Niña & 88 & 48,4 \\
& Niño & 182 & 51,6 \\
Grado & $2-4$ & 114 & 62,6 \\
& $5-8$ & 68 & 37,3 \\
& Público & 118 & 64,8 \\
& Privado & 64 & 35,2 \\
\end{tabular}

La Tabla 2 muestra los datos obtenidos de los padres. Predominó el sexo femenino con un $84,6 \%$. El $46,1 \%$ de los padres está en unión libre, y el rango de edad más frecuente fue entre los 35 a 45 con un $53 \%$. Se evidenció que el $90,7 \%$ de los padres tienen entre 1 a 3 hijos. 
Tabla 2.

Datos sociodemográficos de padres de familia

\begin{tabular}{cccc}
\hline & & FRECUENCIA & PORCENTAJE \\
\hline \multirow{2}{*}{ Sexo } & Mujer & 154 & 84,6 \\
& Hombre & 28 & 15,4 \\
& Soltero & 43 & 23,7 \\
Estado Civil & Casado & 50 & 27,5 \\
& Unión Libre & 84 & 46,1 \\
& Separado & 4 & 2,2 \\
Número de hijos & Viudo & 1 & 0,5 \\
& $1-3$ & 165 & 90,7 \\
& $4-5$ & 17 & 9,3 \\
& $24-34$ & 66 & 36,1 \\
Edad Padres & $35-45$ & 97 & 53 \\
& $46-55$ & 19 & 10,1 \\
& Total & 182 & 100 \\
\hline
\end{tabular}

\subsection{Instrumentos}

\subsubsection{Cuestionario de Estilos Educativos Parentales (CEEP)}

Evalúa rol parental, contextos familiares, estilos educativos, prácticas parentales y competencias emocionales. Es diligenciado por padres con hijos de 6 a 12 años. Consta de 66 ítems, tipo Likert con cuatro opciones de respuesta de 0 (rara vez) a 3 (la mayoría de veces). Los puntajes fueron agrupados en nivel alto, medio y bajo. Su confiabilidad es de 0,92 y sus escalas con 0,64 y 0,84 (Gómez et al., 2013). Para este estudio se empleó la subescala de competencias emocionales adaptada para población colombiana (Moreno Méndez, Espada Sánchez, \& Gómez Becerra, 2019).

\subsubsection{Escala de Autoconcepto de Piers-Harris (1969).}

Mide el nivel de autoconcepto global, por medio de 80 ítems con respuestas dicotómicas ( $\mathrm{Si}$ o No), agrupados en 6 subescalas: físico, social, conductual, intelectual, felicidad-satisfacción y falta de ansiedad en niños entre 7 y 12 años. cuyos puntajes están agrupados en nivel alto, medio y bajo. Está adaptado para población colombiana y cuenta con una confiabilidad general de 0,82 con el coeficiente alfa de Cronbach y de 0,87 en Kuder-Richardson (Guillen \& Ramírez, 2011; Vivas, 1998)

\subsubsection{Lista de Chequeo de la Conducta Infantil (CBCL)}

Formato para padres de Achenbach \& Edelbrock (1983). Consta de 113 ítems que evalúan problemas internalizantes y externalizantes en niños de 5 a 16 años, con opciones de respuestas entre 0 (no es cierto) y 2 (es cierto). Los 
puntajes se agrupan en niveles normal, riesgo y clínico En la adaptación para Colombia, tiene una consistencia interna de 0,94 y una confiabilidad de 0,83 (Hewitt, Jaimes, Vera, \& Villa, 2012; Rescorla, Althoff, Achenbach, \& Ivanova, 2019).

\subsection{Procedimientos desarrollados}

Fase 1. Inicio. Se contactó a los colegios y se enviaron consentimientos, Ley de Habeas Data y asentimientos informados a los participantes con el fin de que conocieran el propósito de la investigación y autorizaran su participación.

Fase 2. Ejecución. Se realizó la aplicación del CEEP y el CBCL formato para padres. Por otro lado, a los niños se les hizo la aplicación de la Escala de Autoconcepto de Piers-Harris dentro de las horas de clase de los colegios, los cuales fueron aplicados por los psicólogos de la presente investigación.

Fase 3. Finalización. Recolección y análisis estadísticos de datos. Adicionalmente, se entrega informe de resultados globales a las directivas de las instituciones y un taller sobre competencias emocionales a padres.

\subsection{Análisis de datos}

Los resultados del estudio se procesaron con el software SPSS, versión 24. Primero, se llevaron a cabo los análisis descriptivos de frecuencia de cada una de las variables. Luego, se realizó la prueba de normalidad de KolmogorovSmirnov. Finalmente, se hicieron los análisis correlacionales entre las variables del estudio. Dado que las variables no presentaron distribución normal, se llevó a cabo el análisis de correlación a través del coeficiente de Spearman.

\section{RESULTADOS}

Los hallazgos que a continuación se presentan se encuentran organizados a nivel descriptivo y a nivel correlacional.

Como se observa en la Tabla 3, al realizar el análisis del Cuestionario de estilos educativos parentales, en la variable de competencias emocionales, los padres de niños de colegios públicos y privados reportaron un nivel medio en los componentes de reconocimiento de emociones, manejo emocional e impulsividad. Esto significa que los padres de familia tienden a identificar las emociones propias y de sus hijos, y ser empáticos con ellos, asimismo, regulan apropiadamente las emociones displacenteras que puedan afectar la interacción con sus hijos. 
Respecto a la competencia de expresión emocional, los hallazgos indican que el $59,3 \%$ de los padres de familia de colegios privados reportaron un nivel bajo, lo cual indica que se encuentran falencias al demostrar afecto, hablar sobre las emociones y reconocerlas entre padres e hijos de forma recíproca. Cabe resaltar que, en los colegios públicos, el $58,4 \%$ de los padres reportaron un nivel alto en esta dimensión, lo cual significa que se presentan demostraciones de afecto y diálogo sobre las emociones en la relación padre-hijo.

Se realizó un análisis por género para identificar los roles parentales en cuanto a las competencias emocionales. Los resultados indican que con respecto al componente de expresión emocional los padres demostraron una menor expresión hacia sus hijos que las madres. En cuanto a la impulsividad, reconocimiento de emociones y manejo emocional se identificó que habían similitudes en los comportamientos de ambos padres hacia sus hijos.

Con respecto a la variable de conductas internalizantes, el CBCL- formato para padres, permite identificar que, los niños de colegios públicos y privados se ubicaron en un rango normal respecto a la clasificación del instrumento; los componentes ansioso/depresivo, retraído/depresivo y quejas somáticas. Dichos datos indican la ausencia de preocupación excesiva, llanto frecuente, sentimientos de tristeza e inferioridad, aislamiento social, timidez; y señalan la ausencia de reportes de malestar físico como dolores de estómago, de cabeza, vómitos sin causa médica, entre otros.

Al analizar la variable de autoconcepto, se evidenció que los niños de ambos tipos de colegio se ubicaron en un rango alto en el componente conductual, un rango bajo para el componente físico y un nivel medio en los componentes de falta de ansiedad, popularidad y felicidad-satisfacción. Los datos anteriores indican que los niños de colegios públicos y privados presentan una autovaloración positiva con relación a su forma de comportarse, teniendo en cuenta el instrumento de autoconcepto aplicado; en términos de expresiones como "me porto bien", "soy obediente". Se perciben de forma negativa en cuanto a sus rasgos físicos, al creer que no son atractivos o les molesta su apariencia. Manifiestan una percepción de bienestar a nivel emocional al considerarse tranquilos y en ausencia de preocupaciones excesivas. Adicionalmente, los hallazgos señalan una buena autovaloración respecto a sus destrezas en la interacción social, y una tendencia a presentar percepciones y sentimientos positivos frente a la vida y la satisfacción con esta.

Por otra parte, con relación al componente intelectual, en los colegios privados el 59,3\% de los participantes reportó un nivel bajo, y en el $50 \%$ de los niños de colegios públicos predominó un rango medio, no obstante, como 
se observa en la Tabla 3, el 48,3 \% de los niños se ubicó en un nivel bajo. Lo anterior señala la existencia de percepciones negativas en ambos tipos de colegios hacia el propio desempeño en tareas académicas e intelectuales.

Tabla 3.

Descriptivos de Competencias emocionales, conductas internalizantes y autoconcepto

\begin{tabular}{|c|c|c|c|c|c|c|c|}
\hline \multirow{3}{*}{$\begin{array}{l}\text { COLEGIO } \\
\text { PRIVADO }\end{array}$} & \multirow[b]{2}{*}{ CEEP } & \multicolumn{6}{|c|}{ RANGO } \\
\hline & & \multicolumn{2}{|c|}{ ALTO } & \multicolumn{2}{|c|}{ MEDIO } & \multicolumn{2}{|c|}{ BAJO } \\
\hline & Competencias emocionales P. & $\mathrm{F}$ & $\%$ & $\mathrm{~F}$ & $\%$ & $\mathrm{~F}$ & $\%$ \\
\hline & Impulsividad & 1 & 1,5 & 45 & 70,3 & 18 & 28,1 \\
\hline & Expresión emocional & - & - & 26 & 40,6 & 38 & 59,3 \\
\hline & Reconocimiento de emociones & - & - & 40 & 62,5 & 24 & 37,5 \\
\hline & Manejo emocional & 11 & 17,1 & 50 & 78,1 & 3 & 4,6 \\
\hline & CBCL & \multicolumn{2}{|c|}{ NORMAL } & \multicolumn{2}{|c|}{ RIESGO } & \multicolumn{2}{|c|}{ CLÍNICO } \\
\hline & Conductas internalizantes & $\mathrm{F}$ & $\%$ & $\mathrm{~F}$ & $\%$ & $\mathrm{~F}$ & $\%$ \\
\hline & Ansioso / depresivo & 56 & 87,5 & 13 & 20,3 & 1 & 1,5 \\
\hline & Retraído / depresivo & 57 & 89 & 14 & 21,8 & 2 & 3,1 \\
\hline & Quejas Somáticas & 59 & 92,1 & 2 & 3,1 & 3 & 4,6 \\
\hline & PIERS Y HARRIS & \multicolumn{2}{|c|}{ ALTO } & \multicolumn{2}{|c|}{ MEDIO } & \multicolumn{2}{|c|}{ BAJO } \\
\hline & Autoconcepto & $\mathrm{F}$ & $\%$ & $\mathrm{~F}$ & $\%$ & $\mathrm{~F}$ & $\%$ \\
\hline & Conductual & 38 & 59,3 & 22 & 34,3 & 4 & 6,2 \\
\hline & Intelectual & - & - & 26 & 40,6 & 38 & 59,3 \\
\hline & Físico & - & - & 23 & 35,9 & 41 & 64 \\
\hline & Falta de ansiedad & 17 & 26,5 & 38 & 59,3 & 9 & 14 \\
\hline & Popularidad & - & - & 58 & 90,6 & 6 & 9,3 \\
\hline & Felicidad-satisfacción & 1 & 1,5 & 60 & 93,7 & 3 & 4,6 \\
\hline & $\mathrm{N}$ & & & & & & \\
\hline \multirow[t]{20}{*}{ PÚBLICO } & CEEP & \multicolumn{2}{|c|}{ ALTO } & \multicolumn{2}{|c|}{ MEDIO } & \multicolumn{2}{|c|}{ BAJO } \\
\hline & Competencias emocionales $P$. & $\mathrm{F}$ & $\%$ & $\mathrm{~F}$ & $\%$ & $\mathrm{~F}$ & $\%$ \\
\hline & Impulsividad & 2 & 1,6 & 71 & 60,1 & 45 & 38,1 \\
\hline & Expresión emocional & 69 & 58,4 & 48 & 40,6 & 1 & 0,84 \\
\hline & Reconocimiento de emociones & 6 & 5 & 93 & 78,8 & 19 & 16,1 \\
\hline & Manejo emocional & 21 & 17,7 & 91 & 77,1 & 6 & 5 \\
\hline & CBCL & \multicolumn{2}{|c|}{ NORMAL } & \multicolumn{2}{|c|}{ RIESGO } & \multicolumn{2}{|c|}{ CLÍNICO } \\
\hline & Conductas internalizantes & $\mathrm{F}$ & $\%$ & $\mathrm{~F}$ & $\%$ & $\mathrm{~F}$ & $\%$ \\
\hline & Ansioso / depresivo & 109 & 92,3 & 9 & 7,6 & - & - \\
\hline & Retraído / depresivo & 105 & 88,9 & 10 & 8,4 & 3 & 2,5 \\
\hline & Quejas Somáticas & 108 & 91,5 & 9 & 7,6 & 1 & 0,84 \\
\hline & PIERS Y HARRIS & \multicolumn{2}{|c|}{ ALTO } & \multicolumn{2}{|c|}{ MEDIO } & \multicolumn{2}{|c|}{ BAJO } \\
\hline & Autoconcepto & $\mathrm{F}$ & $\%$ & $\mathrm{~F}$ & $\%$ & $\mathrm{~F}$ & $\%$ \\
\hline & Conductual & 76 & 64,4 & 41 & 34,7 & 1 & 0,84 \\
\hline & Intelectual & 2 & 1,6 & 59 & 50 & 57 & 48,3 \\
\hline & Físico & 5 & 4,2 & 41 & 34,7 & 72 & 61 \\
\hline & Falta de ansiedad & 28 & 23,7 & 83 & 70,3 & 7 & 5,9 \\
\hline & Popularidad & 10 & 8,4 & 103 & 87,2 & 5 & 4,2 \\
\hline & Felicidad-satisfacción & 1 & 0,84 & 116 & 98,3 & 1 & 0,84 \\
\hline & $\mathrm{N}$ & & & & & & \\
\hline
\end{tabular}


Una vez descritas las variables, se procedió a realizar la prueba KolmogorovSmirnov $(\mathrm{N}>50)$ para determinar si hay normalidad en la distribución de la muestra. En vista de la no normalidad, se aplicó como método estadístico el coeficiente de correlación de rangos de Spearman a las variables competencias emocionales, conductas internalizantes y autoconcepto.

Al realizar el respectivo análisis de correlación entre las tres variables globales, no se encontró relación estadísticamente significativa entre competencias emocionales parentales, conductas internalizantes y autoconcepto en niños. En cuanto a los análisis por componentes, como se observa en la Tabla 4, para la variable de competencias emocionales y su relación con conductas internalizantes se encontró que la impulsividad mantiene una relación positiva con los componentes Ansioso/Depresivo ( $R h o=0,213 p=0,04$ ) y Aislado/Depresivo $(R h o=0,210 ; p=0,004)$. Lo cual significa que la presencia de emociones como la ira en los padres de forma frecuente e injustificada, lo que configuraría una relación maltratante de tipo emocional, podría estar asociada con preocupación excesiva, sentimientos de tristeza, culpa e inferioridad, llanto frecuente y aislamiento social en los niños.

En relación con las competencias emocionales parentales y su asociación con autoconcepto, se evidenció que el manejo emocional mantiene una relación negativa con el componente intelectual del autoconcepto ( $R h o=-0,148 p=0,046)$. Lo cual indica que el adecuado control emocional de los padres, podría estar asociado con una autovaloración negativa en los niños respecto a su desempeño en tareas académicas e intelectuales. Dichos datos sugieren que posiblemente los padres dieron un reporte acorde a lo esperado socialmente en esta dimensión de las competencias emocionales, aspecto que requeriría seguirse evaluando.

Finalmente, para la variable de conductas internalizantes y su relación con el autoconcepto, se encontró que el componente Ansioso/Depresivo mantiene una relación negativa con el componente conductual $(R h o=-0,240 p=0,001)$, físico $(R h o=-0,182 p=0,014)$ y falta de ansiedad del autoconcepto (Rho=-0,213 $p=0,004)$, lo cual significa que la presencia de preocupación excesiva, sentimientos de tristeza y llanto frecuente en los niños podría estar asociada con una autovaloración negativa respecto a su comportamiento, apariencia física y bienestar emocional.

Respecto al componente Aislado/Depresivo, se evidencia que mantiene una relación negativa con los componentes del autoconcepto conductual $(R h o=-0,147$ $p=0,047)$, falta de ansiedad $(R h o=-0,172 p=0,020)$. y popularidad $(R h o=-0,206$ 
$p=0,005)$. Lo cual indica que la presencia de aislamiento social, timidez, preocupación excesiva, sentimientos de tristeza y llanto frecuente en los niños, podría estar asociada con una autovaloración negativa respecto a su forma de comportarse, el propio estado emocional y su desempeño en las relaciones interpersonales.

El componente Quejas Somáticas, mantiene una relación negativa con el componente Falta de ansiedad $(R h o=-0,218 p=0,003)$. Lo cual señala que la autovaloración negativa frente al propio estado emocional, al reportar temor y preocupación de manera excesiva, se encuentra asociada con la presencia de malestar físico como dolores frecuentes de cabeza, estómago, vómitos, entre otros.

Tabla 4.

Correlación entre los componentes de las variables Competencias emocionales, Conductas Internalizantes y Autoconcepto

\begin{tabular}{|c|c|c|c|c|c|c|c|c|c|c|c|c|}
\hline & & & $\begin{array}{l}\text { IMPUL- } \\
\text { SIVI- } \\
\text { DAD } \\
\end{array}$ & $\begin{array}{c}\text { MANEJO } \\
\text { EMOCIO- } \\
\text { NAL }\end{array}$ & $\begin{array}{l}\text { ANSIOSO } \\
\text { - DEPRE- } \\
\text { SIVO }\end{array}$ & $\begin{array}{l}\text { AISLADO } \\
\text { - DEPRE- } \\
\text { SIVO }\end{array}$ & $\begin{array}{c}\text { AUTO } \\
\text { CONDUC- } \\
\text { TUAL }\end{array}$ & $\begin{array}{l}\text { AUTO } \\
\text { FísICO }\end{array}$ & $\begin{array}{l}\text { FALTA } \\
\text { DE AN- } \\
\text { SIEDAD }\end{array}$ & $\begin{array}{c}\text { AUTO } \\
\text { INTELEC- } \\
\text { TUAL } \\
\end{array}$ & $\begin{array}{l}\text { POPU- } \\
\text { LARI- } \\
\text { DAD }\end{array}$ & $\begin{array}{l}\text { FELICI- } \\
\text { DAD }\end{array}$ \\
\hline \multirow{8}{*}{$\begin{array}{c}\text { Rho de } \\
\text { Spearman }\end{array}$} & Impulsividad & $\begin{array}{l}\text { Rho } \\
\text { Sig }\end{array}$ & - & - & $\begin{array}{c}0,213^{* *} \\
0,004\end{array}$ & $\begin{array}{c}0,210^{* *} \\
0,004\end{array}$ & - & - & - & - & - & - \\
\hline & $\begin{array}{l}\text { Manejo } \\
\text { emocional }\end{array}$ & $\begin{array}{l}\text { Rho } \\
\text { Sig }\end{array}$ & - & - & - & - & - & - & - & $\begin{array}{c}-0,148^{*} \\
0,046\end{array}$ & - & - \\
\hline & $\begin{array}{l}\text { Ansioso / } \\
\text { depresivo }\end{array}$ & $\begin{array}{l}\text { Rho } \\
\text { Sig }\end{array}$ & - & - & - & - & $\begin{array}{c}-0,240 * * \\
0,001\end{array}$ & $\begin{array}{c}-0,182^{*} \\
0,014\end{array}$ & $\begin{array}{c}-0,213^{* *} \\
0,004\end{array}$ & - & - & - \\
\hline & $\begin{array}{l}\text { Aislado / } \\
\text { depresivo }\end{array}$ & $\begin{array}{l}\text { Rho } \\
\text { Sig }\end{array}$ & - & - & - & - & $\begin{array}{c}-0,147^{*} \\
0,047\end{array}$ & - & $\begin{array}{c}-0,172^{*} \\
0,020\end{array}$ & - & $\begin{array}{c}-0,206^{* *} \\
0,005\end{array}$ & - \\
\hline & $\begin{array}{c}\text { Quejas } \\
\text { somáticas }\end{array}$ & $\begin{array}{l}\text { Rho } \\
\text { Sig }\end{array}$ & - & - & - & - & - & - & $\begin{array}{c}-0,218^{*} \\
0,003\end{array}$ & - & - & - \\
\hline & $\begin{array}{c}\text { Auto. } \\
\text { Conductual }\end{array}$ & $\begin{array}{l}\text { Rho } \\
\text { Sig }\end{array}$ & - & - & - & - & - & $\begin{array}{c}-0,214^{* *} \\
0,004\end{array}$ & $\begin{array}{c}0,344^{* *} \\
0,000\end{array}$ & - & $\begin{array}{c}0,378^{*} \\
0,000\end{array}$ & - \\
\hline & Auto. Físico & $\begin{array}{l}\text { Rho } \\
\text { Sig }\end{array}$ & - & - & - & - & - & - & $\begin{array}{c}-0,345^{* *} \\
0,000\end{array}$ & - & $\begin{array}{c}0,441^{* *} \\
0,000\end{array}$ & $\begin{array}{c}0,441^{* *} \\
0,000\end{array}$ \\
\hline & $\begin{array}{l}\text { Falta de } \\
\text { Ansiedad }\end{array}$ & $\begin{array}{l}\text { Rho } \\
\text { Sig }\end{array}$ & - & - & $\begin{array}{c}-0,213^{* *} \\
0,004\end{array}$ & $\begin{array}{c}-0,172^{* *} \\
0,020\end{array}$ & - & - & - & - & $\begin{array}{c}0,460 * * \\
0,000\end{array}$ & - \\
\hline
\end{tabular}

\section{DISCUSIÓN}

Con el propósito de establecer la relación entre competencias emocionales de los padres, las conductas internalizantes y la autoestima en niños entre 8 y 12 años de colegios públicos y privados de Bogotá, a continuación se presentan los resultados encontrados.

En cuanto a los descriptivos hallados sobre las competencias emocionales de los padres, dos hallazgos que llaman la atención, fueron los niveles encon- 
trados en los componentes de manejo emocional e impulsividad, los cuales, se presentaron de manera similar en padres de colegios públicos y privados, ubicándose en un rango medio. Estos resultados permiten inferir, que si bien los padres controlan de forma adecuada sus emociones al tratar de no demostrar emociones negativas (ira, enfado, rabia, tristeza, frustración), eventualmente tienden a enfadarse de forma excesiva e injustificada ante comportamientos de sus hijos; lo que concuerda con lo reportado por estudios previos (Altafim, McCoy, \& Linhares, 2018; Barros, Doctor, Peleteiro, Lunet, \& Kana, 2015; Fondo de las Naciones Unidas para la Infancia [UNICEF], 2014), al afirmar que cuando los niños evidencian desobediencia, dificultades para acatar normas y límites, entre otras, los padres tienden a expresar emociones negativas complejas.

Por otro lado, los hallazgos indican que en los padres de niños de colegios públicos, la expresión emocional se ubicó en un rango alto, lo cual señala la presencia significativa de demostraciones de afecto, diálogo sobre las emociones-sentimientos y facilidad para reconocerlos entre padres e hijos de forma recíproca. Cabe resaltar que en el caso de los padres de niños de colegios privados predominó un rango bajo en dicho componente. En coherencia con el nivel medio de impulsividad y lo expuesto por Berger (2007) y Rescorla, Althoff, Ivanova y Achenbach (2019), los padres podrían manifestar ocasionalmente emociones negativas en detrimento de la expresión de afecto.

Con respecto a los niveles normales de las conductas internalizantes reportados en los niños de colegios públicos y privados, difiere de estudios realizados en la ciudad de Bogotá con niños de 8 a 12 años como el de Hewitt, Vila y Juárez (2016), donde se halló un porcentaje mayor de niños ubicados en un rango clínico. Esto podría explicarse por la presencia de factores protectores en el entorno más próximo de los niños que conformaron la muestra (Hoyos, Lemos \& Torres de Galvis, 2012). Estos pueden ser variables familiares, como la cohesión familiar (Bronfenbrenner \& Morris, 2006; Starr \& Dávila, 2009), variables individuales como los altos niveles de autoconcepto en la mayoría de sus componentes, los cuales, asociados a las prácticas parentales positivas, podrían conducir a una menor presencia de problemas de ajuste psicológico en los niños (Fuentes, García, Gracia \& Alarcón, 2015).

Conviene resaltar la posibilidad de que los niveles normales obtenidos en las conductas internalizantes también estén relacionados con la dificultad para identificar este tipo de comportamientos por parte de los padres. Según Lecannelier (2018), la dimensión internalizante es más difícil de detectar, ya que el niño inhibe sus emociones sin expresarlas hacia el medio. De acuerdo 
con ello, resulta pertinente en futuras investigaciones tomar en cuenta el reporte de los niños.

Con respecto a la variable de autoconcepto, uno de los hallazgos más significativos, fueron los altos niveles en el componente conductual, lo cual es coherente con los resultados expuestos por Cayetano Poma (2012) y MorenoMéndez et al. (2011), quienes encontraron que los niños tienden a tener una percepción positiva sobre su forma de comportarse.

Para el componente intelectual del autoconcepto, se pudo evidenciar que en los colegios privados predominó un rango bajo. Lo cual difiere de los hallazgos de Urquijo (2002), quien encuentra un nivel alto en dicho componente. Sin embargo, es posible que los niños de colegios privados puedan tener una percepción negativa frente a sus capacidades intelectuales o habilidades escolares, en función a variables como las metodologías de enseñanza y la presión académica, en términos de la cantidad y calidad de exigencias académicas.

Por otra parte, al encontrar una percepción negativa en la mayoría de los participantes frente a su imagen corporal, se difiere con el estudio de Moreno-Méndez, et al. (2011), al encontrar que la mayoría de los estudiantes de colegios públicos reportan un nivel alto. Para el presente estudio dichos niveles en ambos tipos de colegios, podrían indicar que los niños posiblemente no aceptan su cuerpo con sus limitaciones y defectos; y no reciben aprobación, o refuerzo social (González, Núñez, Glez \& García, 1997).

Respecto a la relación hallada entre la impulsividad de los padres y los componentes ansioso-depresivo y retraído-depresivo, concuerda con Cerezo, Ruiz-Esteban, Sánchez-Lacasa y Arense-Gonzalo (2018), Milojevich y Haskett (2018), Mirabile (2014) y Moreno Méndez, Espada Sánchez y Gómez Becerra (2020), en cuyos estudios encontraron relaciones positivas entre las reacciones punitivas y la internalización de estados emocionales desadaptativos, las cuales son más fuertes en altos niveles de expresión de emociones negativas por parte de los padres. Existe la posibilidad de que la asociación encontrada entre dichas variables esté mediada por aspectos como los conflictos parentales al interior del hogar y el estrés parental como lo reportan Cummings y Merrilees (2010) y Lee, Pace, Lee y Knauer (2018) lo cual sería pertinente profundizar en próximos estudios.

Richaud de Minzi (2009) manifiesta que, en las etapas iniciales en el desarrollo de los niños, se puede identificar cómo el núcleo familiar se 
convierte en la base donde se aprenden y constituyen las primeras reglas de comportamiento a través del modelado. En función a ello, un mecanismo que explica la presencia frecuente de ira de forma inconsistente e injustificada en los padres y su asociación con los componentes ansioso-depresivo y retraído-depresivo, puede ser el procesamiento cognitivo de los padres, específicamente la tendencia a reevaluar o no situaciones estresantes. Según Morris, Silk, Steinberg, Myers y Robinson (2007), aquellos padres que lo hacen, llegan a estar más tranquilos y reflexivos al relacionarse con sus hijos, y por consiguiente modelan un comportamiento apropiado para el control de las emociones en los niños.

Respecto a la relación entre las competencias emocionales y el autoconcepto, los resultados indican que el componente de manejo emocional se encuentra asociado negativamente al autoconcepto intelectual. Dicha relación es contraria a lo reportado por la evidencia acumulada (Ajilchi, et al, 2013; Paulus, Licata, Gniewosz, \& Sodian, 2018). A su vez podría estar asociada a la interpretación que los padres hicieron de la dirección de los ítems del instrumento, aspecto que es importante seguir investigando.

En lo referente a las variables conductas internalizantes y autoconcepto, se concuerda con Mazadiego, Vera y Ruiz (2011) al reportar que una autovaloración positiva o negativa por parte de los niños puede estar relacionada con problemas de tipo internalizante o externalizante, tales como la ansiedad, depresión, abuso de sustancias, conductas delictivas, entre otras. Adicionalmente, Brand, Yoeli, Gilboa, Benaroya y Apter (2015), en su estudio encontraron que altos niveles de depresión se asociaron con una baja autopercepción del niño.

Frente a los hallazgos obtenidos, se puede afirmar que los bajos niveles de autoconcepto en los niños podrían actuar como variable mediadora temprana en la presencia de problemas comportamentales y afectivos. Hayden, Olino, Mackrell, Jordan, Desjardins y Katsiroumbas (2013), sugieren que los estilos de vulnerabilidad cognitiva representan un factor de riesgo para la depresión en los niños; por lo cual la cognición negativa puede ser importante en los esfuerzos de prevención. Adicionalmente, se ha encontrado que factores como la autoeficacia tienen un poder predictor de aspectos comportamentales, afectivos y de afrontamiento (Lightsey, Maxwell, Nash, Rarey, \& McKinney, 2011).

Al considerar que las asociaciones encontradas en los componentes de las variables fueron débiles, una posible explicación es el hecho de no haber 
contado con muestras clínicas y el tamaño muestral con el que se trabajó. No obstante, las relaciones significativas apoyan la hipótesis correlacional, que si bien no se acerca a la causalidad puede proporcionar información útil a nivel teórico, social y clínico.

Los datos encontrados en la presente investigación apoyan la importancia de trabajar en educación emocional, teniendo en cuenta que los padres actúan como agentes modeladores. Bisquerra (2003), Viloria (2005) y Fernández y Montero (2016) mencionan cómo la educación emocional debe ser transversal a la vida del niño en todos sus contextos y encaminarse al entendimiento de las propias emociones y la de los demás. Los resultados pueden ser un insumo en el desarrollo de estrategias en pro de fomentar y fortalecer competencias emocionales en los padres, que resulten adecuadas en el marco de la crianza para prevenir el maltrato infantil y contribuir al bienestar socioemocional de los niños, favoreciendo el adecuado establecimiento de relaciones interpersonales, la sana convivencia y ajuste en las áreas social, personal, académico y familiar. Adicionalmente, es fundamental afianzar en los niños con problemas internalizantes, competencias orientadas a sobrevivir, prosperar, perseverar, conciliar e integrar experiencias traumáticas en el desarrollo saludable de la identidad y abogar por sí mismos (Yoon, Dillard, Pei, Shockley, McCarthy, Beaujolais, Wang, Maguire-Jack, Wolf, \& Cochey, 2020), que los hagan más resilientes cuando hayan experimentado situaciones estresantes como resultados de una crianza maltratante.

Cabe resaltar que los hallazgos encontrados sirven de insumo en el contexto educativo, debido a que resaltan la importancia de fortalecer el autoconcepto y su trascendencia en el desarrollo óptimo a nivel físico, psicológico y social. Moreno-Méndez, et al. (2011) mencionan que se puede lograr esto mediante espacios de comunicación y convivencia donde se trabaje la motivación del niño y la familia como factor protector, se fomente el diálogo en la resolución de conflictos, la toma de decisiones, la expresión y regulación de emociones, entre otros.

Para finalizar, es importante resaltar que el presente estudio tuvo ciertas limitaciones debido al alcance y tiempo de duración de la investigación, por un lado, no se tuvo en cuenta el género para identificar posibles diferencias entre niños y niñas, así como madres y padres en la socialización de competencias emocionales como lo refieren Sánchez, Fernández-Berrocal, Montañés y Latorre (2008). A su vez, no se tuvo en consideración el estrato socioeconómico de los padres, y como lo mencionan Prooijen, et al. (2018) este factor puede influir en las pautas de crianza, la presencia de comporta- 
mientos internalizantes y la baja percepción del autoconcepto de los niños. Adicionalmente, otra de las limitaciones fue la falta de homogeneidad en el número de participantes de colegios públicos y privados.

Se recomienda para futuras investigaciones considerar las limitaciones presentadas para obtener resultados más precisos sobre las correlaciones entre las variables estudiadas. Adicionalmente, dada la mayor participación de madres en la presente investigación, resulta pertinente tener en cuenta para próximos estudios, si dichas diferencias entre los roles paternos respecto al componente de expresión emocional llegan a ser significativas en una muestra más homogénea. Como lo mencionan Yap, Morgan, Cairns, Jorm, Hetrick \& Merry (2016), se ha producido un aumento en el rol del padre como agente modelador en el manejo, socialización y reconocimiento de emociones.

Adicionalmente, se recomienda indagar sobre otras variables relacionadas al desarrollo de conductas internalizantes en niños, tales como, la violencia intrafamiliar y el trauma infantil; como lo menciona Vanegas (2014), los niños que han experimentado situaciones de maltrato por parte de sus padres dentro del hogar comúnmente se mantienen ansiosos, tímidos y deprimidos. La autora también menciona que la sintomatología internalizante se relaciona con una vulnerabilidad en el niño a desarrollar estrés postraumático en situaciones complicadas del cotidiano.

\section{CONCLUSIONES}

Teniendo en cuenta los objetivos del presente estudio, el análisis presentado permite concluir que entre las competencias emocionales parentales, conductas internalizantes y autoconcepto a nivel general no se evidencia una relación estadísticamente significativa. Sin embargo, con respecto a los objetivos específicos se encontraron correlaciones estadísticamente significativas $(R h o<0,30)$ (Cohen, 1988 ) entre sus componentes ansioso-depresivo, aislado depresivo e impulsividad en los padres.

De acuerdo a lo anterior, es posible afirmar que la forma en que gestionan y expresan los padres sus emociones representa un pilar importante para favorecer la salud mental de sus hijos. La falta o deficiencia de competencias emocionales en el marco de la crianza, que incluye la presencia frecuente e inconsistente de reacciones de ira excesivas e injustificadas de los padres, puede estar asociada con la forma en que los niños afrontan distintas situaciones vitales y con la probabilidad de que se generen dificultades a nivel 
afectivo y comportamental, como la preocupación excesiva, sentimientos de inferioridad, tristeza y aislamiento social en los hijos.

Por otro lado, se concluye que la presencia de conductas asociadas con la ansiedad, depresión o quejas psicosomáticas, no solo están relacionadas con la impulsividad de los padres, sino también, con la forma en que los hijos se perciben así mismos en algunas dimensiones, entre ellas su estado emocional, desempeño social, forma de comportarse en distintos contextos donde se desenvuelven y la manera en que se valoran físicamente.

Con el fin de disminuir la presencia de problemas de tipo internalizante y autoconcepto de los niños, entre otras problemáticas que se podrían derivar a largo plazo como lo es el abandono escolar, dicha información sirve de soporte para el desarrollo e implementación de iniciativas de intervención orientadas al fortalecimiento de las competencias emocionales parentales para contribuir al bienestar socioemocional de los niños y adolescentes.

Conflicto de interés: No se declaran conflictos de interés por parte de los autores.

Agradecimientos: Los autores agradecen a los participantes y a las instituciones educativas el apoyo recibido para llevar a cabo el estudio.

Financiamiento: El manuscrito es resultado del trabajo de grado llevado a cabo por los autores financiado por la Facultad de Psicología de la Universidad San Buenaventura Colombia en la ciudad de Bogotá.

\section{REFERENCIAS}

Achenbach, T. M., \& Edelbrock, C. S. (1983). Manual of Child Behavior Checklist and Revised Child Behavior Profile. Burlington: University of Vermont, Department of Psychiatry.

Ajilchi, B., Kargar, F. R., \& Ghoreishi, M. K. (2013). Relationship of the parenting styles of overstressed mothers with the anxiety of their children. Procedia-Social and Behavioral Sciences, 82, 520-524. https://doi.org/10.1016/j.sbspro.2013.06.302

Altafim, E. R. P., McCoy, D. C., \& Linhares, M. B. M. (2018). Relations between parenting practices, socioeconomic status, and child behavior in Brazil. Children and Youth Services Review, 89, 93-102. https://doi.org/10.1016/j.childyouth.2018.04.025

Ato, M., López, J., \& Benavente, A. (2013). Un sistema de clasificación de los diseños de investigación en psicología. Anales de Psicología, 29(3), 1038-1059. http:// www.redalyc.org/articulo.oa?id=16728244043

Barros, H., Doctor, H. V., Peleteiro, B., Lunet, N., \& Kana, M. A. (2015). Maternal and child health interventions in Nigeria: a systematic review of published studies from 1990 to 2014. BMC Public Health, 15(1), 334. https://doi.org/10.1186/ s12889-015-1688-3. 
Berger, K. (2007). Psicología del desarrollo: infancia y adolescencia. En K. Stassen Berger, \& K. Berger, El Juego: los padres (p. 704). Madrid: Editorial Médica Panamericana.

Bisquerra, R. A. (2003). Educación emocional y competencias básicas para la vida. Revista de Investigación Educativa, 21(1), 7-43. http://revistas.um.es/rie/article/ view/99071

Brand, A., Yoeli, N., Gilboa, E., Benaroya, N., \& Apter, A. (2015). Perceptions of self, mother and family and behavior of prepubertal depressed children. European Psychiatry, 30(1), 69-74. https://doi.org/ 10.1016/j.eurpsy.2014.05.005

Bronfenbrenner, U., \& Morris, P. (2006). The bioecological model of human development. theoretical models of human development. https://doi. org/10.1002/9780470147658.chpsy0114

Cayetano Poma, N. (2012). Autoestima en estudiantes de sexto grado de primaria de instituciones educativas estatales y particulares de Carmen de la Legua-Callao (Trabajo de grado). Loyola. http://repositorio.usil.edu.pe/ handle/123456789/1131

Cerezo, F., Ruiz-Esteban, C., Sánchez-Lacasa, C., \& Arense-Gonzalo, J. J. (2018). Dimensions of parenting styles, social climate, and bullying victims in primary and secondary education. Psicothema, 30(1), 59-65. https://doi.org/10.7334/ psicothema2016.360

Cohen, J. (1988). Statistical power analysis for the behavioral sciences. Routledge.

Cuartas, J., Ward, K. P., Ma, J., \& Grogan-Kaylor, A. (2020). Physical punishment and Colombian children and adolescents' cognitive and behavioral outcomes. Journal of Applied Developmental Psychology, 68, 101140. https://doi.org/10.1016/j. appdev.2020.101140

Cummings, E. M., \& Merrilees, C. E. (2010). Identifying the dynamic processes underlying links between marital conflict and child adjustment In M. S. Schulz, M. K. Pruett, P. K. Kerig, \& R. D. Parke (Eds.), Decade of behavior (science conference). Strengthening couple relationships for optimal child development: Lessons from research and intervention (pp.27-40). American Psychological Association. https://doi.org/10.1037/12058-003

Denham, S. A., Bassett, H. H. \& Wyatt, T. (2007). The socialization of emotional competence. In J. E. Grusec, \& P. D. Hastings (Eds.), Handbook of Socialization, 614-637. New York: The Guilford Press.

Doan, S. N., Son, H., \& Kim, L. N. (2018). Maternal and paternal emotional contributions to children's distress tolerance: Relations to child depressive symptoms. Psychiatry Research. https://doi.org/ 10.1016/j.psychres.2018.06.008

Esnaola, I., \& Goñi, A., \& Madariaga, J. (2008). El autoconcepto: Perspectivas de investigación. Revista de Psicodidáctica, 13(1), 69-96. http://www.redalyc.org/ articulo.oa?id=17513105

Fernández, A. M., \& Montero, I. (2016). Aportes para la educación de la inteligencia emocional desde la educación infantil. Revista Latinoamericana de Ciencias Sociales, Niñez Y Juventud, 14(1), 53-66. https://doi.org/10.11600/169 2715x.1412120415

Fondo de las Naciones Unidas para la Infancia (UNICEF). (2014). Ocultos a plena luz. Un análisis estadístico de la violencia contra los niños. http://www.unicef.org/ ecuador/ocultos_a_plena_luz.pdf+\&cd=1\&hl=es\&ct=clnk\&gl=pe

Fuentes, M. C., García, F., Gracia, E., \& Alarcón, A. (2015). Los estilos parentales de socialización y el ajuste psicológico. Un estudio con adolescentes españoles. Revista de Psicodidáctica, 20(1), 117-138. https://doi.org/10.1387/RevPsicodidact.10876 
Franco, N., Pérez, M., \& De Dios, M. (2014). Relación entre los estilos de crianza parental y el desarrollo de ansiedad y conductas disruptivas en niños de 3 a 6 años. Revista de Psicología Clínica con Niños y Adolescentes, 1(2), 149-156. https://dialnet.unirioja.es/servlet/articulo?codigo $=4742071$

García, M., Espinosa, J., Jiménez, F., \& Parra, J. (2013). Separados y desiguales. Educación y clases sociales en Colombia. Bogotá, D.C.: Ediciones Antropos.

Gómez, I. B., García-Barranco, M. R., De las Heras, R. R., Martínez de Salazar, A. A., Cobos, L. S., Martín, M. J. G., \& Fernández, C. S. (2013). Estudio piloto de validación del cuestionario de evaluación de estilos educativos parentales-memoria del proyecto de investigación. Comunicación Invitada en el II Symposium Nacional de Psicología Clínica y de la Salud con Niños y Adolescentes. Elche (Alicante).

González, J., Núñez, J., Glez, S. \& García, M. (1997). Autoconcepto, autoestima y aprendizaje escolar. Revista Psicothema, 9(2), 271-289. http://www.psicothema. com/psicothema.asp?id=97

Guillen, F., \& Ramírez, M. (2011). Relación entre autoconcepto y condición física en alumnos de tercer ciclo de primaria. Revista de Psicología del Deporte, 20, 45-59. http://www.redalyc.org/articulo.oa?id=235119302005

Hayden, E. P., Olino, T. M., Mackrell, S. V., Jordan, P. L., Desjardins, J., \& Katsiroumbas, P. (2013). Cognitive vulnerability to depression during middle childhood: Stability and associations with maternal affective styles and parental depression. Personality and individual differences, 55(8), 892-897. https://doi.org/10.1016/j. paid.2013.07.016

Hewitt, N., Jaimes, S., Vera., L. A., \& Villa, M. C. (2012). Características psicométricas del cuestionario de comportamientos infantiles $C B C L$ en niños y adolescentes colombianos. (Trabajo de Grado). Universidad de San Buenaventura, Bogotá - Colombia. http://www.scielo.org.co/scielo.php?script=sci_nlinks\&re$\mathrm{f}=000133 \&$ pid $=\mathrm{S} 0123-9155201400010000900022 \& \operatorname{lng}=e n$

Hewitt, N., Vila, J., \& Juárez, F. (2016). Predicción de los problemas de banda ancha y banda estrecha mediante los modelos transaccional, cognitivo social y de regulación emocional. (Tesis Doctoral) Granada: Universidad de Granada. http:// hdl.handle.net/10481/42435

Hewitt-Ramírez, N., \& Moreno-Méndez, J. (2018). Intervención psicológica para comportamientos externalizados e internalizados en niños de 8 a 12 años. Revista de Psicológica Clínica con Niños y Adolescentes, 5(2), 37-42. https://doi. org/10.21134/rpcna.2018-05.2.5

Hincapié, L., Montoya., D., \& Dussan, C. (2016). Caracterización del autoconcepto en niños y niñas en situación de desplazamiento en Mocoa, Colombia. Revista de Investigación UCM, 16(27), 118-132. https://dialnet.unirioja.es/servlet/articulo?codigo $=6045672$

Hoyos, E., Lemos, M., \& Torres de Galvis, Y. (2012). Factores de riesgo y de protección de la depresión en los adolescentes de la ciudad de Medellín. International Journal of Psychological Research, 5(1), 109-121. https://biblat.unam.mx/hevila/ Internationaljournalofpsychologicalresearch/2012/vol5/no1/12.pdf

Jadue J, G. (2003). Transformaciones familiares en Chile: Riesgo creciente para el desarrollo emocional, psicosocial y la educación de los hijos. Estudios Pedagógicos (Valdivia), 29, 115-126. https://dx.doi.org/10.4067/S0718-07052003000100008

Jiménez, D. (2015). Percepción filial sobre los estilos familiares en menores con ideas suicidas (Tesis doctoral). Universidad Jaume I, España. http://repositori.uji.es/ xmlui/bitstream/handle/10234/129365/TFG_2014_vergeD.pdf 
Lee, S. J., Pace, G. T., Lee, J. Y., \& Knauer, H. (2018). The association of fathers' parental warmth and parenting stress to child behavior problems. Children and Youth Services Review, 91, 1-10. https://doi.org/10.1016/j.childyouth.2018.05.020

Leathers, S. J., Spielfogel, J. E., Geiger, J., Barnett, J., \& Voort, B. L. V. (2019). Placement disruption in foster care: Children's behavior, foster parent support, and parenting experiences. Child Abuse \& Neglect, 91, 147-159. https://doi.org/10.1016/j. chiabu.2019.03.012

Lecannelier, F. (2018). El trauma oculto en la infancia. Guía científicamente informada para padres, educadores y profesionales. Penguin Random House.

Lightsey, O., Maxwell, D., Nash, T., Rarey, E., \& McKinney, V. (2011). Self-control and self-efficacy for affect regulation as moderators of the negative affect-life satisfaction. relationship. Journal of Cognitive Psychotherapy, 25(2), 142-153. https:// doi.org/10.1891/0889-8391.25.2.142

López, M. J. R., Quintana, J. C. M., Casimiro, E. C., \& Chávez, M. L. M. (2009). Las competencias parentales en contextos de riesgo psicosocial. Psychosocial Intervention, 18(2), 113-120. http://www.redalyc.org/pdf/1798/179814021003.pdf

López Soler C., Alcántara, M. V., Fernández, V., Castro, M., \& López Pina, J. A. (2010). Características y prevalencia de los problemas de ansiedad, depresión y quejas somáticas en una muestra clínica infantil de 8 a 12 años, mediante el CBCL (Child Behavior Checklist). Anales de Psicología, 26, 325-334. http://www.redalyc.org/ articulo.oa?id=16713079017

Martínez, M., \& García, M. C. (2012). La crianza como objeto de estudio actual desde el modelo transaccional. Revista Latinoamericana de Ciencias Sociales, Niñez y Juventud, 10(1), 169-178. https://dialnet.unirioja.es/descarga/articulo/3933513. pdf

Mazadiego-Infante, T., Vera-Pedroza, A., \& Ruiz-Carús, S. (2011). Problemas internalizados y externalizados en una muestra de niños de Educación Básica. Journal of Behavior, Health \& Social Issues, 3(1), 17-23 https://doi.org/10.5460/jbhsi. v3.1.27680

Milojevich, H. M., \& Haskett, M. E. (2018). Longitudinal associations between physically abusive parents' emotional expressiveness and children's self-regulation. Child Abuse \& Neglect, 77, 144-154. https://doi.org/ 10.1016/j.chiabu.2018.01.011

Ministerio de Salud Mental (2017). Boletín de salud mental en niños, niñas y adolescentes. Número 4. https://www.minsalud.gov.co/sites/rid/Lists/BibliotecaDigital/ RIDE/VS/PP/ENT/boletin-4-salud-mental-nna-2017.pdf

Mirabile, S. P. (2014). Parents' inconsistent emotion socialization and children's socioemotional adjustment. Journal of Applied Developmental Psychology, 35(5), 392-400. https://doi.org/ 10.1016/j.appdev.2014.06.003

Moreno Méndez, J. H., Espada Sánchez, J. P., \& Gómez Becerra, I. (2019). Advances on parental educational styles predictors of internalizing and externalizing behaviors in children. Universitas Psychologica, 18(5), 1-10. https://doi.org/10.11144/ Javeriana.upsy18-5.apes

Moreno Méndez, J. H., Espada Sánchez, J. P., \& Gómez Becerra, M. I. (2020). Role of parenting styles in internalizing, externalizing, and adjustment problems in children. Salud Mental, 43(2), 73-84. https://doi.org/10.17711/SM.01853325.2020.011

Moreno Méndez, J. H., Muñoz, Á. Á., Sánchez, B. C., Triana, P. C., Chemas, N. L., \& Barón, A. M. (2011). Autoestima en un grupo de niños de 8 a 11 años de un colegio público de la ciudad de Bogotá. Psychologia: Avances de la Disciplina, 5(2), 155-162. https://www.redalyc.org/articulo.oa?id=297224105013 
Morris, A. S., Silk, J. S., Steinberg, L., Myers, S. S., \& Robinson, L. R. (2007). The role of the family context in the development of emotion regulation. Social Development, 16(2), 361-388. https://doi.org/10.1111/j.1467-9507.2007.00389.x

Organización Mundial de la Salud (2017). Reporte sobre la salud mental: Depresión. http://www.who.int/mental_health/management/depression/es/

Ortiz, M. J., Sánchez, F. L., Rebollo, M. J. F., \& Etxebarria, I. (2014). Desarrollo afectivo y social. Ediciones Pirámide.

Paulus, M., Licata, M., Gniewosz, B., \& Sodian, B. (2018). The impact of motherchild interaction quality and cognitive abilities on children's self-concept and self-esteem. Cognitive Development, 48, 42-51. https://doi.org/10.1016/j. cogdev.2018.07.001

Piers, E. V., \& Harris, D.B. (1969). The Piers-Harris Children's Self-Concept Scale. Nashville. Tennesse: Counselor Recording and Tests.

Posada, A. (2013).Lasaludmentalen Colombia.Biomédica, 33(4), 497-498. http://www. scielo.org.co/scielo.php?script=sci_arttext\&pid=S0120-41572013000400001\&In$\mathrm{g}=\mathrm{en} \& \mathrm{t} \operatorname{lng}=\mathrm{es}$

Prooijen, D., Hutteman, R., Mulder, H., \& Laceulle, O. (2018). Self-control, parenting, and problem behavior in early childhood: A multi-method, multi-informant study. Infant Behavior and Development, 50, 28-41. https://doi.org/10.1016/j. infbeh.2017.11.001

Ramírez, M. A. (2005). Padres y desarrollo de los hijos: prácticas de crianza. Estudios Pedagógicos, 31(2), 167-177. https://doi.org/10.4067/S0718-07052005000200011

Rescorla, L. A., Althoff, R. R., Achenbach, T. M., \& Ivanova, M. Y. (2019). Effects of society and culture on parent's ratings of children's mental health problems in 45 societies. European Child \& Adolescent Psychiatry. https://doi.org/10.1007 / s00787-018-01268-3

Restrepo, J., \& Rivera, J. (2017). Práctica de taekwondo y comportamientos internalizantes y externalizantes en niños con edades entre los 6 y los 12 años. Revista Katharsis, 23, 2-17. http://revistas.iue.edu.co/index.php/katharsis/article/view/862

Richaud de Minzi, M. C. (2009). Influencia del modelado de los padres sobre el desarrollo del razonamiento prosocial en los/las Niños/as. Revista Interamericana de Psicología, 43(1), 187-198. http://pepsic.bvsalud.org/scielo.php?scrip$\mathrm{t}=\mathrm{sci}$ arttext\&pid=S0034-96902009000100021

Ruvalcaba, N., Gallegos, J., Flores, A., \& Fulquez, S. (2013). Las competencias socioemocionales como factor protector ante la sintomatología de ansiedad y depresión en adolescentes. Psicogente, 16(29), 55-64. http://revistas.unisimon. edu.co/index.php/psicogente/article/view/1941

Sánchez, M., Fernández-Berrocal, P., Montañés, J., \& Latorre, J. (2008). ¿Es la inteligencia emocional una cuestión de género? Socialización de las competencias emocionales en hombres y mujeres y sus implicaciones. Revista Electrónica de Investigación Psicoeducativa, 15(6), 455-474. https://dialnet.unirioja.es/servlet/ articulo?codigo $=2855720$

Sher-Censor, E., Shulman, C., \& Cohen, E. (2018). Associations among mothers' representations of their relationship with their toddlers, maternal parenting stress, and toddlers' internalizing and externalizing behaviors. Infant Behavior and Development, 50, 132-139. https://doi.org/ 10.1016/j.infbeh.2017.12.005

Starr, L., \& Dávila, J. (2009). Differentiating interpersonal correlates of depressive symptoms and social anxiety in adolescence: implications for models of comorbidity. Journal of Clinical Child \& Adolescent Psychology, 37(2), 337-349. https:// doi.org/10.1080/15374410801955854 
Symeou, M., \& Georgiou, S. (2017). Externalizing and internalizing behaviours in adolescence, and the importance of parental behavioural and psychological control practices. Journal of Adolescence, 60, 104-113. https://doi.org/10.1016/j. adolescence.2017.07.007

Ternera, L. A. C. (2014). El desarrollo del autoconcepto en niños y niñas y su relación con la interacción social en la infancia. Psicogente, 17(31), 67-69. https://doi. org/10.17081/psico.17.31.1470

Turcule, A., \& Tulbure, C. (2014). The relation between the emotional intelligence of parents and children. Procedia-Social and Behavioral Sciences, 142, 592-596. https://doi.org/10.1016/j.sbspro.2014.07.671

Urquijo, S. (2002). Auto-concepto y desempeño académico en adolescentes: relaciones con sexo, edad e institución. PsicoUSF, 7(2), 211-218. https://doi. org/10.1590/S1413-82712002000200010.

Vanegas, B. (2014). Violencia intrafamiliar y problemas de conducta internalizada externalizada en niños y niñas escolarizados de La Guajira - Colombia. Cultura, Educación y Sociedad, 5(1), 137-148. https://revistascientificas.cuc.edu.co/culturaeducacionysociedad/article/view/1000

Viloria, C. de A. (2005). La educación emocional en edades tempranas y el interés de su aplicación en la escuela. Programas de educación emocional, nuevo reto en la formación de los profesores. Tendencias Pedagógicas, 10, 107-123. https:// revistas.uam.es/tendenciaspedagogicas/article/view/1856

Vinacur, T. (2015). ¿Los alumnos de las escuelas privadas están mejor preparados para ingresar a la universidad? Revista Colombiana de Educación, 70, 175-200. http://www.scielo.org.co/pdf/rcde/n70/n70a09.pdf

Vivas, J. (1998). Adaptación de la escala de autoconcepto Piers y Harris para la ciudadanía de Bogotá. Bogotá: Grujel Ltda.

Yoon, S., Dillard, R., Pei, F., Shockley, K., McCarthy, K., Beaujolais, B., Wang, X., Maguire-Jack, K., Wolf, K., \& Cochey, S. (2020). Defining resilience in maltreated children from the practitioners' perspectives: A qualitative study. Child Abuse \& Neglect, 106. 104516. https://doi.org/10.1016/j.chiabu.2020.104516

Yap, M., Morgan, A., Cairns, K., Jorm, A., Hetrick, S., \& Merry, S. (2016). Parents in prevention: A meta-analysis of randomized controlled trials of parenting interventions to prevent internalizing problems in children from birth to age 18. Clinical Psychology Review, 50, 138-158. https://doi.org/10.1016/j.cpr.2016.10.00

Esta obra está bajo: Creative commons attribution 4.0 international license. El beneficiario de la licencia tiene el derecho de copiar, distribuir, exhibir y representar la obra y hacer obras derivadas siempre y cuando reconozca y cite la obra de la forma especificada por el autor o el licenciante.

\section{(cc) BY}

